\section{Felix Klein und das Riemannsche Erbe} von Reinhold Remmert

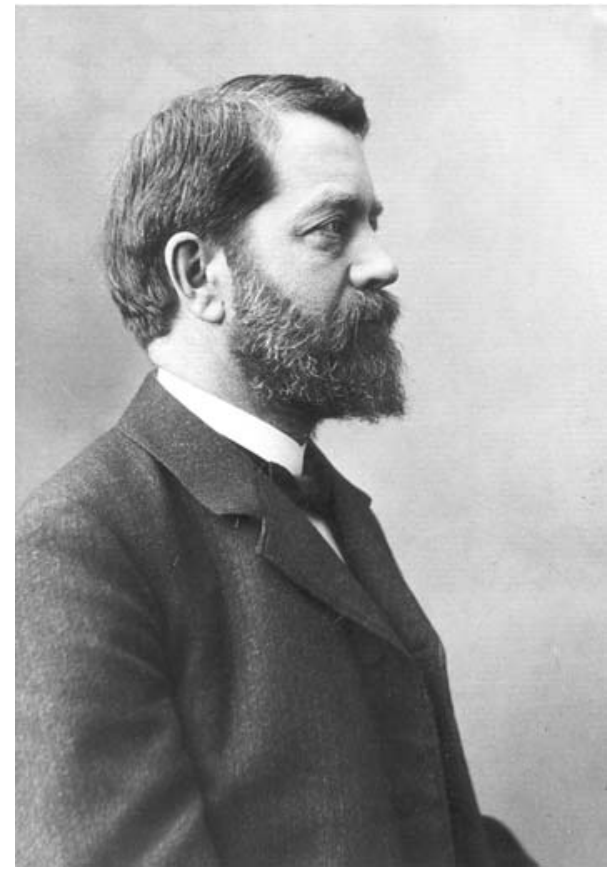

Felix Klein (Quelle: Universität Göttingen)

\begin{abstract}
Mit leichtem Herzklopfen trete ich vor dieses Auditorium, um über einen Mathematiker der Georgia Augusta zu sprechen, der vor 150 Jahren - fast auf den Tag genau - geboren wurde und der heute eine Legende ist. Mit Herzklopfen, denn über Felix Klein ist schon viel gesagt und geschrieben worden, wenngleich die ihm in all seinen Facetten gerecht werdende Biographie noch fehlt. Ich werde versuchen, nicht nur ein Panegyriker zu sein.
\end{abstract}

Am 3. Dezember 1929 weihte die Göttinger Mathematische Gesellschaft in einer Festsitzung an der Bunsenstraße das neue Mathematische Institut ein. Es war von Klein ersonnen und begonnen, immer wieder durchdacht und auf vielen Wegen gefördert. Man feierte die Krönung seines Werks, dessen Vollendung er nicht mehr erlebte. Die Festrede hielt Hermann Weyl: Felix Kleins Stellung in der mathematischen Gegenwart [W1930]. Weyl stand in seiner Göttinger Zeit (1904-1913) in nahem Kontakt zu Klein. Er widmete ihm 1913 sein Jugendwerk Die Idee der Riemannschen Fläche ,in Dankbarkeit und Vereehrung"; er lehnte aber 1920 die Berufung zum KleinNachfolger ab. Weyl, Meister des geschliffenen Wortes, dem nach eigenem Bekunden ,fast mehr an Ausdruck und Gestaltung [lag] als an der Erkenntnis selbst" [W1955, S. 653], verführt geradezu zum Zitieren. Dies werde ich im folgenden besonders gerne tun.

\section{Höhepunkte der Kleinschen Mathematik}

Leopold von Ranke notiert 1877 als 82-jähriger in seinem Tagebuch: „Musiker und Mathematiker ha- ben das Vorrecht, in frühen Jahren etwas Vollendetes leisten zu können." Felix Klein mit seiner überquellenden Fruchtbarkeit bis zu seinem 33sten Lebensjahr ist ein herausragendes Beispiel. Weyl vergleicht Kleins wissenschaftliche Laufbahn mit der Bahn einer Rakete: „Sie steigt glänzend, steil zusammengefaßt, empor: die Jahre 1869 bis 1882, die ihn in einem außerordentlich intensiven und ergebnisreichen Forschen zeigen. Dann [ab 1882] beginnt sie sich plötzlich breit zu entfalten und ihren leuchtenden Segen, rückwärts gekrümmt, über das Land auszuschütten: Es entstehen die großen zusammenfassenden Darstellungen des von ihm Errungenen, seine praktischorganisatorische Tätigkeit setzt in großem Stil ein. Der Wendepunkt ist eine Katastrophe, die den 33jährigen trifft, der Zusammenbruch seiner Gesundheit Ostern 1882. ... Nur mit Bewunderung und Ehrfurcht können wir auf das gewaltige Werk schauen, mit dem dieser im Innersten getroffene Mann dann in den folgenden Jahren doch noch die Welt beschenkt hat." [W1930, S. 296]

Ich werde nicht den mächtigen und ideenreichen Organisator zeichnen, der fruchtbringend für die Herausführung der Mathematik aus ihrer kulturellen Isolierung wirkte, der sich für ihre Verknüpfung mit den

Ergänzte Fassung eines am 29. April 1999 vor der Mathematischen Gesellschaft in Göttingen gehaltenen Vortrags 
Anwendungen in Physik und Technik einsetzte und der für das Bürgerrecht der Schulmathematik an den Universitäten stritt. Ich werde auch nicht die ,machtvolle Persönlichkeit eines Olympiers mit dem Willen zur Tat" schildern, für dessen letzten Jahre H. Kneser die Worte fand, [Kn1949a, S. 413]: „Milde Altersweisheit verklärte sein Wesen, die Herrscherzüge waren zurückgetreten, der Humor fehlte nicht."

Schon gar nicht werde ich den Mann darstellen, „der als wissenschaftlicher Führer ... eine oft und heiß umstrittene Erscheinung gewesen" ist und bei dem, wie der 1933 aus Deutschland vertriebene Richard Courant 1926 weiter sagt [C1926, S. 3], „das Wichtigste, was der große Führer braucht, nicht fehlte: Der Geist des Dienens und der Unterordnung unter die Sache, die große Naivität der Sachlichkeit, die weder Rücksicht auf die eigene noch die fremde Person kennt." [Courant stellt auch die Frage: „Kann und darf es in der freien Wissenschaft Führerwillen und Führergewalt geben?", verfolgt sie aber nicht.] Eine prophetische Selbstanalyse seiner „Herrschernatur" (so Max Liebermann) gab Klein schon 1872 in einem Brief an Lie vom 3. August, als das Schicksal ihn nach Erlangen verschlagen hatte: „Der in mir ziemlich stark ausgeprägte sociale Trieb, wenn ich darunter die Lust verstehe, auf andere Menschen zu wirken, wird also voraussichtlich seine Befriedigung finden."

Ich werde vielmehr versuchen, Ihnen den Mathematiker Klein nahe zu bringen, ,der einer ganzen Epoche das Siegel seines Genius aufdrückte" [W1930, S. 299], und der lebendig und mächtig in die mathematische Gegenwart ausstrahlt. Die großen Jahre des Forschens liegen vor seiner (zweiten) Göttinger Zeit. Edelsteine seines Schaffens sind: Ab 1868: Liniengeometrie; 1872: Erlanger Programm; 1874-91: freiere Auffassung von Riemannschen Flächen (Atlasprinzip); ab 1874: automorphe Funktionen; 1882: Grenzkreistheorem (Kronjuwel); 1884: Ikosaederbuch.

Ich stelle die Funktionentheorie in den Mittelpunkt.

\section{Riemanns Vermächtnis}

Am Anfang war Bernhard Riemann (1826-1866). Mit seiner Dissertation beginnt 1851 hier zu Göttungen eine stille Revolution. Riemann lichtet mit seinen Flächen den Nebel um die mehrdeutigen Funktionen, die durch eine Art von Divination - ,in hehrer Einsamkeit, um sie kein Ort" - mehrblättrig über seiner Zahlenkugel schweben (Überlagerungsprinzip). Riemann skizziert ein großes Programm, ,zur Erläuterung unserer allgemeinen Sätze [wird jedoch] ein ausgeführtes Beispiel ihrer Anwendung von Nutzen sein" [R, S. 39]. Dieses (einzige) Beispiel in der Dissertation ist sein Abbildungssatz, S. 40; aquila non captat muscas. 1857 wird er in seinem Meisterwerk
Abel'sche Functionen konkreter. Jetzt ist sein Credo allgegenwärtig: Riemannsche Flächen waren schon, ehe es Funktionen gab. Er erklärt aus der Vogelperspektive der (noch nicht entwickelten) Analysis Situs die inneren Zusammenhänge zwischen algebraischen Funktionen und ihren Integralen auf kompakten Riemannschen Flächen. Die Zahl $p$, von Alfred Clebsch (1833-1872) 1865 treffend das Geschlecht der Fläche genannt, durchzieht wie ein Sauerteig Riemanns Meditationen.

Riemann, ,zu dem wir Nachgeborenen wie zu einem Heiligen aufblicken" [K1926, S. 247], blieb lange unverstanden. Schon 1864 klagte Clebsch in einem Brief an Gustav Roch (1839-1866), der Riemanns Ungleichung zu einer Gleichung, dem seither unsterblichen Satz von Riemann-Roch, veredelte: „[Ich habe] von Riemann's Abhandlung nur sehr wenig verstanden." Und Alexander Brill (1842-1935) und Max Noether (1844-1921) urteilen 1894 in ihrem der Deutschen Mathematiker Vereinigung erstatteten Bericht [BN1894, S. 266]: „In ihrer knappen Darstellungsform, ihrer Gedankenfülle und Tiefe war diese Arbeit für die mathematische Welt ... ein Buch mit sieben Siegeln." Im gleichen Jahr schreibt Klein [K III, S. 490]: „Die Riemannschen Methoden waren damals noch eine Art Arcanum seiner direkten Schüler und wurden von den übrigen Mathematikern fast mit Mißtrauen betrachtet." Erste Versuche, Riemanns Vermächtnis der mathematischen Welt vorzustellen, machten 1865 Carl Gottfried Neumann (1832-1925) sowie 1866 Clebsch und Paul Gordan (1837-1912) mit ihren Büchern Vorlesungen über Riemann's Theorie der Abelschen Integrale bzw. Theorie der Abelschen Functionen. Doch sie blieben Rufer in der Wüste. Der erfolgreiche Evangelist wurde Felix Klein, für Richard Courant (18881972) „der leidenschaftlichste ... Apostel des Riemannschen Geistes" [C1925, S. 202].

\section{Kleins Weg zu Riemann - Mathematische Physik}

In den späten sechziger Jahren wurde Klein hier in Göttingen (als post doc) von „meinem Lehrer Clebsch auf die Riemannschen Resultate auf das Nachdrücklichste hingewiesen" [K III, S. 477]. Indessen bin ich ,erst von 1872 an, als ich schon in Erlangen war, allmählich in Riemanns Ideen eingedrungen" [K1926, S. 266]. Seinen Weg in Riemanns schwer zugängliche Gedankenwelt bahnt er sich über die ihn faszinierende Zusammenhangszahl $p$. Deren Verständnis „war für mich ein geradezu quälendes Problem“ [K II], S. 5]. Seine ersten Arbeiten zu Riemannschen Flächen aus den Jahren 1874-76 machen Bemerkungen über den Zusammenhang der Flächen [K II, 63 ff.]. 
Doch dieser „Kontakt mit dem Riemannschen Ideenkreise [war] nur erst ein vorläufiger. ... Ich habe den Übergang zu dem, was ich den ,echten' Riemann zu nennen pflege, also die Benutzung des Riemannschen Existenztheorems unmittelbar von der Fläche aus, erst von 1877 an vollzogen" [K II, S. 5]. Er meint hier seine physikalische Ableitung des fundamentalen Existenzsatzes für Potentialfunktionen, bei der „die Riemannsche Fläche ... als gleichförmig elektrisch leitend gedacht [wird]. Das läßst sich sehr einfach realisieren, indem man die Fläche mit Stanniol beklebt. . . In zwei Punkten $A_{1}, A_{2}$ werden die Pole einer galvanischen Batterie... aufgesetzt. Es entwickelt sich ein Strom, dessen Potential $u$ auf der Fläche [nur] in $\mathrm{A}_{1}, \mathrm{~A}_{2} \ldots$ unstetig wird wie $\log r_{1}$ bzw. $-\log r_{2}$ " $[\mathrm{K}$ 1926, S. 260]. Diese Potentialfunktionen hat er dann, ,statt immer wieder [über ihre Existenz] zu grübeln, frischweg [benutzt]" [K 1926, S. 266], sie liefern in der Tat alles, was das Herz begehrt. Klein hatte keine Hemmungen, seine ,physikalischen Anschauungen geradezu zum Ausgangspunkt meiner Darstellung zu machen. Riemann verwendet statt ihrer ... das Dirichletsche Prinzip. Aber ich kann nicht zweifeln, daß er von analogen physikalischen Problemen ausgegangen ist, und ihnen nur hinterher, um die physikalische Evidenz durch einen mathematischen Schluß zu stützen, das Dirichletsche Prinzip substituiert hat" [K III, S. 502]. Klein scheint davon überzeugt gewesen zu sein, daß die ,grundlegenden Ideen in Riemanns Phantasie auf Grund der anschaulich-physikalischen Denkweise entstanden sein mögen."

Dieser kühne Zugang zu den Existenzsätzen ist durch James Clerk Maxwell (1831-1879) motiviert. Klein „nimmt die physikalische Analogie zum hodegetischen [=hinführendem] Prinzip" [K III, S. 503], und sucht „durch physikalische Erwägungen zu einer wirklichen Beherrschung der Grundgedanken der Riemannschen Theorie zu gelangen ... Ich habe meine Schrift über Riemann geradezu als Physiker geschrieben, unbekümmert um alle die vorsichtigen Zusätze, die man bei ausgeführter mathematischer Behandlung zu machen gewohnt ist" [K III, S. 478]. Arnold Sommerfeld (1868-1951) meint in [S. 1919, S. 301]: „Es ist nicht eigentlich mathematische Physik, was hier getrieben wird, sondern physikalische Mathematik ... Die Physik ... beflügelt den mathematischen Gedanken." Aber Klein ist kein Physiker. Zu keiner Zeit hat er den mathematischen Beweis als das endgültige Kriterium entthronen wollen. „Im Zentrum seiner wissenschaftlichen Persönlichkeit war Klein reiner Mathematiker und ist es stets geblieben." [W1930, S. 293]

Kleins Ziel ist Klarheit der Erkenntnis, formale Exaktheit und präzise Details sind seine Sache nicht.,,Unstrenge in der Form“ nimmt er in Kauf.
„Ich würde produciren können, wenn mir Einzelkeiten nicht zu gleichgültig wären," klagt er in einem Brief an Lie vom 28. 6. 1873. Seine Stärke war das intuitive, die Zusammenhänge erschauende Verstehen. Er „war eine durchaus visuelle Natur und als solche völlig unmusikalisch in so hohem Maße, daß ihn das Klavierüben seiner Töchter bei der Arbeit nicht störte." [S. 1949, S. 289]

Kleins Ausführungen über Riemann sind auch für seine eigene Art aufschlussreich: „Gewiß ist es der Schlußstein am Gebäude einer jeden mathematischen Theorie, den zwingenden Beweis für alle Behauptungen zu erbringen. Gewiß spricht sich die Mathematik selbst das Urteil, wenn sie auf zwingende Beweise verzichtet. Das Geheimnis genialer Produktivität wird es ewig bleiben, neue Fragestellungen zu finden, neue Theoreme zu ahnen, die wertvolle Resultate und Zusammenhänge erschließen. Ohne die Schaffung neuer Gesichtspunkte, ohne die Aufstellung neuer Ziele, würde die Mathematik in der Strenge ihrer logischen Beweisführung sich bald erschöpfen und zu stagnieren beginnen, indem ihr der Stoff ausgehen möchte. - So ist die Mathematik in gewissem Sinne von denen am meisten gefördert worden, die mehr durch Intuition als strenge Beweisführung sich auszeichneten. Es ist kein Zweifel, daß Riemann derjenige Mathematiker der letzten Dezennien ist, der heute noch am lebendigsten nachwirkt" [K1926, S. $271 / 72$ ]. Schon früh stellt Klein sich mit Leidenschaft gegen die in Berlin gängige Meinung, daß Riemannsche Flächen lediglich ein „Versinnlichungsmittel" - man konzedierte: ein sehr wertvolles, sehr suggestives - beim Studium mehrdeutiger Funktionen sind. Bereits 1882 betont er [K III S. 555]: „Die Riemannsche Fläche [veranschaulicht] nicht nur die in Betracht kommenden Funktionen, sondern sie definiert dieselben."

Courant wertet 1926 in seiner Gedächtnisrede [C1926, S. 7]: „Es [war] eine gewaltige Leistung des jungen Felix Klein, die Schätze des ,echten' Riemannschen Genius zu heben. Eine Leistung, die nur einem congenialen Geiste möglich war, der unbekümmert um die Forderungen der Mode seinen Weg geht." Weyl urteilt vier Jahre später [W1930, S. 299]: „Klein hat die Grundideen der Riemannschen Funktionentheorie, geleitet von suggestiven physikalischen Anschauungen, in befreiter und schöpferischer Weise zur Geltung gebracht."

\section{Riemannsche Flächen bei Klein - Atlasprinzip}

Von der Mitte der siebziger Jahre an beginnt Klein, sich von der Vorstellung zu befreien, daß Riemannsche Flächen a priori konkret über der Kugel schwe- 


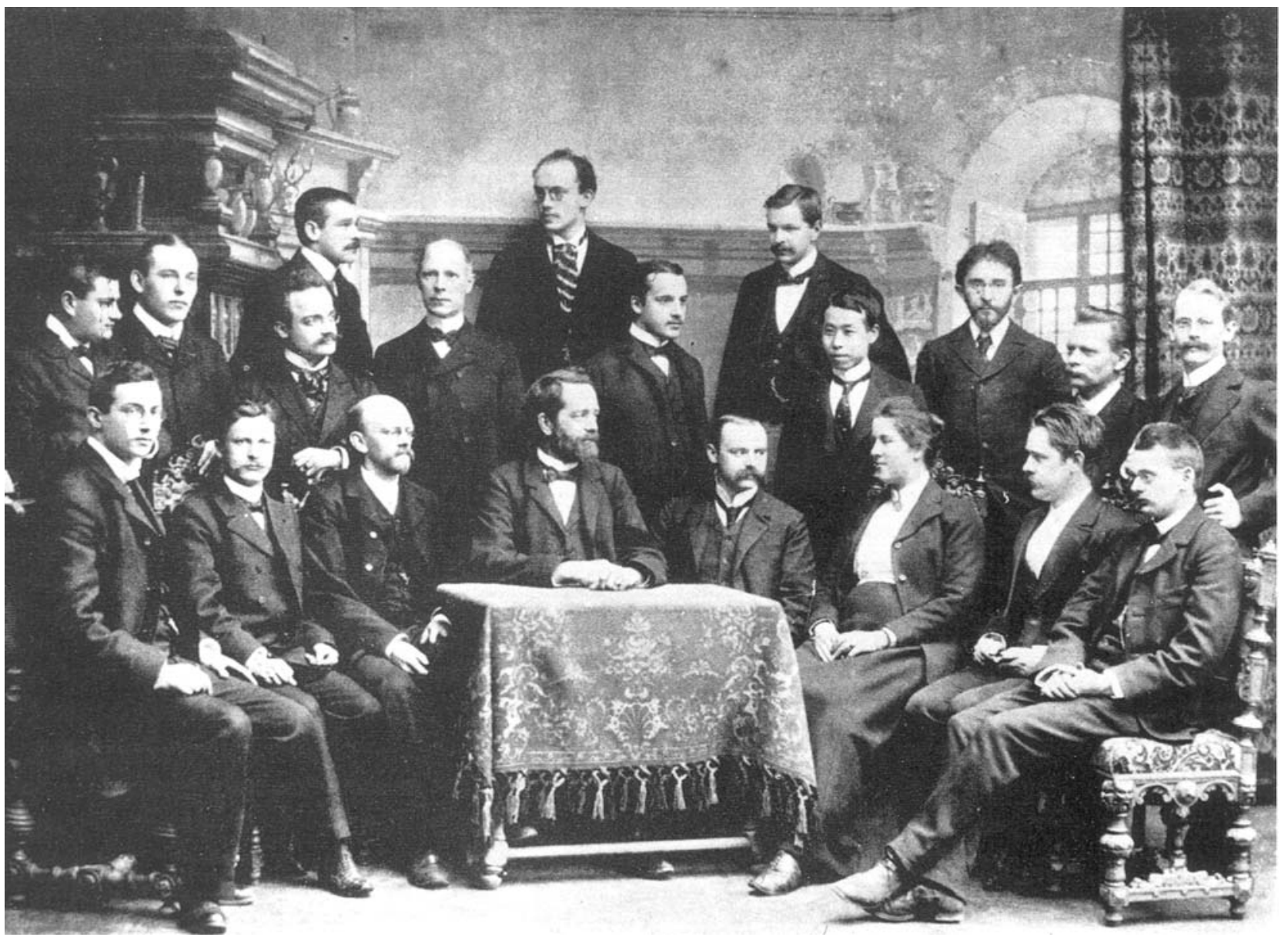

Felix Klein (Quelle: Universität Göttingen)

ben. In dem „,kleinen Büchlein“ Über Riemanns Theorie der algebraischen Funktionen und ihrer Integrale [K III, S. 499-573], das aus seinen Leipziger Vorlesungen [K1880/81] über Riemannsche Flächen hervorgegangen ist, berichtet er 1882 in der Vorrede, S. 501/502, über ein Gespräch mit dem RiemannSchüler Friedrich Emil Prym (1841-1915) aus dem Jahre 1874: „Er erzählte mir, daß die Riemannschen Flächen ursprünglich durchaus nicht notwendig mehrblättrige Flächen über der Ebene sind, daß man vielmehr auf beliebig gegebenen krummen Flächen ganz ebenso komplexe Funktionen des Ortes studieren kann." Doch vierzig Jahre später revoziert Klein: Prym habe ihm 1882 geschrieben, daß er sich nicht mehr genau an seine Bemerkung erinnere, daß er aber niemals den Gedanken gehegt habe, „daß bei Riemann die Untersuchung von Funktionen auf beliebigen Flächen der Untersuchung der Funktionen in einer über der Ebene ausgebreiteten mehrblättrigen Fläche vorausgegangen sei“ [K III, S. 479]. Dies ist wohl ein einmaliger Fall, wo eine große Idee auf eine Bemerkung zurückgeht, an die sich der Redner nicht erinnern kann und die der Zuhörer falsch verstanden hat.
Klein diskutiert seinen neuen kühnen Zugang zu Riemannschen Flächen erstmals 1882. In [K III, S. 520], betrachtet er auf einer beliebigen krummen [reellanalytischen] Fläche $X \mathrm{im} \mathbb{R}^{3}$, die mit der induzierten Riemannschen Metrik $d s^{2}=E d p^{2}+2 F d p d q+G d q^{2}$ versehen ist, die Geschwindigkeitspotentiale u stationärer Ströme. Sie genügen der Beltramischen Differentialgleichung

$$
\frac{\partial\left(\left(F \frac{\partial u}{\partial q}-G \frac{\partial u}{\partial p}\right) / H\right)}{\partial p}+\frac{\partial\left(\left(F \frac{\partial u}{\partial p}-E \frac{\partial u}{\partial q}\right) / H\right)}{\partial q}=0
$$

$\left(H:=\sqrt{E G-F^{2}}\right)$; im Fall $X=\mathbb{R}^{2}$ hat man $E=G=H=1, F=0$, und gerade die klassische Potentialgleichung $\Delta u=0$.

$\mathrm{Zu} u$ existiert ein konjugiertes Potential $v$, die „,komplexe Funktion $u+i v$ des Ortes auf der Fläche" ist unter konformen Abbildungen von $X$ invariant. Die Fläche wird durch $x \mapsto u(x)+i v(x)$ im Kleinen bikonform auf ein Gebiet in $\mathbb{C}$ abgebildet, daher lassen sich holomorphe Funktionen auf $X$ lokal invariant definieren. In heutiger Sprache hat Klein der Fläche lokal eine von der Metrik herrührende (kanonische) holomorphe Struktur gegeben, vgl. hierzu auch [KIII, 


\section{S. 480] und S. 634.}

In seinen Göttinger Vorlesungen [K1891/92, S. 22 ff.], wird Klein allgemeiner. Er ersetzt die Fläche im $\mathbb{R}^{3}$ durch eine ,zweidimensionale geschlossene Mannigfaltigkeit, auf welcher irgendein definiter Differentialausdruck $d s^{2}$ vorgegeben ist. Ob diese Mannigfaltigkeit in einem Raum von 3 oder mehr Dimensionen gelegen ist, das ist nun dabei ganz gleichgültig“" Er erkennt, daß bei orientierbaren Flächen konforme Strukturen nur lokal nötig sind, um den Schritt vom Kleinen ins Große zu tun; er sagt das so, S. 26:

Eine zweidimensionale, geschlossene, mit einem Bogenelement $d s^{2}$ ausgestattete Mannigfaltigkeit (welche keine Doppelmannigfaltigkeit ist [=welche orientierbar ist]) ist jedenfalls dann als Riemannsche Mannigfaltigkeit [=Fläche] zu brauchen, wenn man sie mit einer endlichen Zahl von Bereichen dachziegelartig überdecken kann, deren jedes eindeutig und konform auf eine schlichte Kreisscheibe abgebildet werden kann.

Da im Überlappungsbereich von Dachziegeln wegen der Orientiertheit der Fläche stets dieselbe konforme Struktur entsteht (Gruppeneigenschaft), so braucht Klein keine Verträglichkeitsbedingungen für Dachziegelabbildungen. Aus Kleins Dachziegelüberlegungen wurden holomorphe Atlanten. Klein bereitete den Weg zur Definition komplexer Mannigfaltigkeiten mittels des Atlasprinzips. Seit langem ist sein Vorgehen nun Folklore, er aber klagt noch 1923 in [K III, S. 479], daß „die Idee ... wenig Nachahmung gefunden [hat]". Ausnahmen scheinen ihm lediglich 1898 H. Poincarés kurze Abhandlung Les fonctions fuchsiennes et l'équation $\Delta u=e^{u}$ [P II, 67-70], sowie - man liest es und staunt - H. Weyls Buch Die Idee der Riemannschen Fläche (1913) zu machen.

Klein neigte nicht zum abstrakten Denken. Der Axiomatik als Instrument der Forschung stand er ablehnend gegenüber; Weyl schreibt [W1930, S. 293]: „Er betonte gerne, daß die Erkenntnis sozusagen in der Mitte beginnt und nicht nur nach oben, sondern auch nach unten $\mathrm{zu}$ sich ins Ungewisse verliert. Unsere Aufgabe ist es, in beiden Richtungen allmählich das Dunkel zurückzudrängen; das absolute Fundament aber, der große Elephant, der den Turm der Wahrheit auf seinem sicheren Rücken trägt, sei wohl nur ein Märchen."

\section{Transformationsgruppen}

Während seiner akademischen Wanderjahre ist Klein 1870 mit seinem Freund Sophus Marius Lie (18421899) in Paris. Dort war gerade Camille Jordans Traité des Substitutions erschienen. Dieses Buch erschlieft beiden eine neue mathematische Welt. Als
Klein nach nur 21/2 Monaten Paris infolge des Kriegsausbruchs verlassen muß, trägt er, wie Courant [C1925, S. 199], formulierte, den Stein der Weisen mit sich. Von Stund' an benutzte er den Gruppenbegriff "als ordnendes Prinzip im Wirrsal der Erscheinungen" [K III, S. 3]. Schon zwei Jahre später definiert er im „Erlanger Programm" Geometrie als Invariantentheorie zu einer Transformationsgruppe. Am 12. Dezember 1873 schreibt er an Lie: „Ich bin ein Transformationsmensch."

Gruppen sind für Klein zeitlebens Transformationsgruppen, die auf einem Raum wirken (verwirklicht sind). Die Degradierung der Transformation zu Elementen völlig gleichgültiger Natur, wo man beim Rechnen ,nur aufzupassen [hat], daß man nicht gegen die aufgestellten vier Gebote [Gruppenaxiome] verstößt," machte er sich nicht zu eigen. Bei dieser „abgeblaßten Definition“... wird das logische Skelett sorgfältig herauspräpariert, [doch] der Appell an die Phantasie tritt völlig zurück.“ ..., Überhaupt hat die Methode den Nachteil, daß sie nicht zum Denken anregt" [K1926, 335-36]. Würde heute sein Urteil milder ausfallen?

\section{Automorphe Funktionen}

Auch die Funktionentheorie unterstellt Klein bald dem Regiment der Gruppe. Wichtige klassische Funktionen wie die Exponentialfunktion und die elliptischen Funktionen sind gegenüber einer unendlichen Gruppe von Translationen invariant. Klein kehrt das Denkschema um: Gegeben sei eine Gruppe, bestimme alle unter ihr invarianten Funktionen. Er beginnt 1874 mit endlichen Gruppen gebrochen linearer Substitutionen auf der Zahlenkugel und ihren invarianten rationalen Funktionen. An Lie schreibt er am 30. April 1874: „Ich halte mich für ziemlich sicher, daß alle endlichen Gruppen linearer Transformationen ... durch die Bewegungen repräsentiert werden, welche einen [der Kugel einschriebenen] regulären [= platonischen] Körper in sich überführen." Und 1922 erinnert er sich: „Die Resultate zeigten, daß ich eine erzführende Ader angeschlagen hatte" [K II, S. 255].

Er beschäftigt sich 1874 auch bereits mit unendlichen diskontinuierlichen Gruppen. Unabhängig von Dedekind (1877) gelangt er 1878 zur absoluten Invarianten der Modulgruppe $\Gamma:=S L_{2}(\mathbb{Z})$, der $J$-Funktion

$$
J:=g_{2}^{3} / \Delta \text { mit } \Delta:=g_{2}^{3}-27 g_{3}^{2}[\text { K III, S. 15]. }
$$

Schnell schließt er Kongruenzuntergruppen $\Gamma_{n} \triangleleft \Gamma$, die Normalteiler von endlichem Index sind, mit ein. Er interessiert sich für die Riemannsche Fläche $X_{n}$, die durch Kompaktifizieren des Quotienten $\mathbb{H} / \Gamma_{n}$ entsteht. ( $\mathbb{H}:=$ obere Halbebene). Auf $X_{n}$ operiert die endliche Gruppe $\Gamma / \Gamma_{n}$ holomorph. Die „Kleinsche 
Fläche" $X_{7}$ vom Geschlecht 3 mit ihren 168 Automorphismen (ternäre Gruppe) ist heute ein Denkmal, ihr Fundamentalbereich in Form eines symmetrischen 14-Ecks wird noch immer bewundert [K III, S. 126].

In seiner Münchner Vorlesung vom Sommer 1879 greift er erneut sein Leitproblem auf: „Alle diskontinuierlichen Gruppen einer Variablen, bzw. die zugehörigen automorphen Funktionen aufzustellen" [K III, S. 581]. Funktionentheorie und Gruppentheorie vereinigen sich nun zu einem mächtigen Strom. Die Tendenz ist „Verschmelzung von Galois und Riemann" [K1926, 366-67].

In jener Münchner Zeit wird Gordan in Erlangen sein „eifrigster Mitarbeiter“. Man trifft sich sonntags zumeist in Eichstätt, weil in der Mitte von Erlangen und München gelegen. Rückschauend nennt Klein diese Jahre, die Gordan später als die Epoche der „mathesis quercupolitana" (quercus = Eiche) apostrophierte, ,die glücklichste Periode meiner mathematischen Produktion" [K II, S. 259]. In seinen Lebenserinnerungen [K1923, S. 21], sagt er: „Die Darstellung der Gesamttheorie, wie ich sie geplant hatte, wurde später von Robert Fricke in langjähriger Arbeit durchgeführt und in den beiden Werken über elliptische Funktionen und automorphe Funktionen niedergelegt.

Hiermit führte ich eine wissenschaftliche Arbeitsweise ein, an der ich von dieser Zeit an festgehalten habe: Ich beschränkte mich auf Ideen und Richtlinien und überließ die genaue Durchführung und weitere Ausgestaltung den jüngeren Kräften, die mir helfend zur Seite standen." Fricke hat dieses Unternehmen mit leidenschaftlichem Eifer erfolgreich durchgeführt, schon bald war er viel mehr als ein Sherpa. Die Bände von Klein-Fricke sind bis heute - aller Weitschweifigkeit zum Trotz - ein Standardwerk geblieben. R. Godement nannte sie allerdings einmal „ouvrage classique monumentale illisible."

\section{Agon mit Poincaré - Grenzkreistheorem}

Unter Grenzkreistheorem versteht man den Uniformisierungssatz für algebraische Funktionen vom Geschlecht $>1$. Es ist der klassische Gipfelpunkt der Theorie der automorphen Funktionen zu diskontinuierlichen Gruppen im Einheitskreis. Bis 1881 ist Klein unangefochten der Meister und Verkünder dieser gruppentheoretischen Funktionentheorie. Er kennt die gesamte Literatur über spezielle diskontinuierliche Gruppen, namentlich die Untersuchungen von Riemann, Schwarz, Fuchs, Dedekind und Schottky. Da schreckt ihn 1881 eine Comptes-Rendus-Note eines ihm bis dato unbekannten Henri Poincaré (18541912) von der französischen Provinzuniversität Caen auf, [P II], 1-4. Dieser umreißt auf 31/2 Seiten ein Programm, das neue, Epoche machende Impulse auslöste: Studium endlich erzeugter diskontinuierlicher Gruppen $G$ von im Einheitskreis $\mathbb{E}$ linearen Transformationen und der $G$-automorphen Funktionen mittels Quotienten von Poincaréschen $\Theta$-Reihen

$$
\begin{aligned}
\Theta(z):= & \sum_{g \in G} H(g z)\left(\frac{d g}{d z}\right)^{m}= \\
& \sum_{\nu=1}^{\infty} H\left(\frac{a_{\nu} z+b_{\nu}}{c_{\nu} z+d_{\nu}}\right)\left(c_{\nu} z+d_{\nu}\right)^{-2 m}, m \geq 2,
\end{aligned}
$$

wo $H$ rational und polfrei auf $\partial \mathbb{E}$ ist. Damit entstehen auf einen Schlag viele neue kompakte Riemannsche Flächen $\mathbb{E} / G$ mit interessanten meromorphen Funktionen.

Nun beginnt ein Agon, den der Jüngere gewinnt. Er kennt zwar kaum die vorhandene Literatur, z. B. hat er noch nie etwas vom Dirichletschen Prinzip gehört, doch er hat seine $\Theta$-Reihen (noch am 7. Mai 1882 fragt Klein Poincaré, wie er die Konvergenz seiner Reihen beweist [K III, S. 612]). Poincaré hat wohl sofort die Uniformisierung aller algebraischen Kurven angestrebt. Dieudonné, [D1975, S. 53], meint sogar, daß Klein erst zum Grenzkreistheorem fand, als er spürte, daß Poincaré dieses Ziel verfolge.

In einer am 27. März 1882 bei den Mathematischen Annalen eingereichten 21/2-seitigen Mitteilung formuliert Klein sein Grenzkreistheorem [K III, S. 627]:

Unter allen auf einer [algebraischen] Riemannschen Fläche [vom Geschlecht> 1] existirenden unverzweigten Functionen giebt es immer eine und [von gebrochen linearen Transformationen abgesehen] nur eine $\eta$-Funktion der zweiten Art. Alle anderen unverzweigten Functionen sind eindeutige Functionen dieses $\eta$.

Bei Poincaré liest sich das zwei Wochen später in einer am 10. April 1882 eingereichten 21/2-seitigen Comptes-Rendus-Note so [P II, 41-42]:

Soit $f(x, y)=0$ une relation algébrique. Il existera, en général, deux fonctions fuchsiennes $F(z)$ et $F_{1}(z)$, jouissant des propriétés suivantes :

$1^{\circ}$ Elles n'existent qu'à l'intérieur du cercle fondamental.

$2^{\circ}$ Si l'on fait $x=F(z), y=F_{1}(z)$, la relation $f(x, y)=0$ est satisfaite.

Den Beweis führen Klein und Poincaré mit einer Kontinuitätsmethode. Sie mußte damals vage bleiben, da die allgemeinen topologischen Begriffe und Sätze fehlten. Klein hat die Unzulänglichkeiten der Kontinuitätsmethode zeitlebens nicht verwunden. Noch 1923 versuchte er in [K III, S. 731] eine Rechtfertigung: 
„Die Theoreme ... sind alle in vollem Umfange bestätigt worden ...; dabei gelangte auch mein Kontinuitätsbeweis zu vollständig einwandfreier Duchführung." Doch hier irrte Klein. Die Schwierigkeiten wurden erst in den fünfziger Jahren durch Ahlfors und Bers in der Theorie der Teichmüllerräume überwunden, vgl. [Pa1997, 237-238].

Klein erklimmt mit dem Grenzkreistheorem den höchsten Gipfel der Riemannschen Funktionentheorie. Er hat es „mit einer divinatorischen Kraft ohnegleichen erschaut und hingestellt, in der Durchführung aber schwang Poincaré sich über ihn hinaus" [W1930, S. 295].

Es ist interessant zu lesen, wie Poincaré und Klein ihre Sternstunden erlebt haben. Poincaré berichtet in Science et méthode, [P1908, S. $50 \mathrm{ff}$.$] , wie ihm mit-$ ten in fremdartiger Umgebung die Erleuchtung kam, daß die biholomorphen Abbildungen des Einheitskreises bzw. der oberen Halbebene auf sich Isometrien der nicht-euklidischen Geometrie sind: „Als ich gerade den Fuß auf das Trittbrett setzte, kam mir, ohne daß meine Gedanken irgendwie darauf vorbereitet waren, die Idee, daß die Transformationen, welche ich zur Definition der Fuchsschen Funktionen benutzte, mit den Bewegungen der nichteuklidischen Geometrie identisch seien. Ich hatte keine Zeit, das zu verifizieren, denn im Omnibus beteiligte ich mich wieder an der begonnenen Konversation, und doch hatte ich sofort volle Gewißheit. Nach meiner Rückkehr nach Caen verifizierte ich das Resultat zur Beruhigung meines Gewissens."

Klein schildert sein Schicksalsjahr 1882 in [K III, S. 584]: „Ostern 1882 war ich zur Erholung meiner Gesundheit an die Nordsee gereist. ... Ich habe es dort aber nur acht Tage lang ausgehalten, denn die Existenz war zu kümmerlich, da ... sich bei mir starkes Asthma einstellte. ... In der letzten Nacht vom 22. zum 23. März, die ich wegen Asthmas auf dem Sofa sitzend zubrachte, stand plötzlich um $21 / 2$ Uhr das Grenzkreistheorem, wie es durch die Figur des Vierzehnecks in Bd. 14 der Math. Annalen ja eigentlich schon vorgebildet war, vor mir. Am folgenden Vormittag in dem Postwagen ... durchdachte ich das, was ich gefunden hatte, noch einmal bis in alle Einzelheiten. Jetzt wußte ich, daß ich ein großes Theorem gefunden hatte." Und in [K1926, S. 380] bekennt er: „Der Preis, den ich für meine Arbeit habe zahlen müssen, war allerdings außerordentlich hoch, er war, daß meine Gesundheit vollends zusammenbrach ... Meine eigentliche produktive Tätigkeit auf dem Gebiet der theoretischen Mathematik ist 1882 zugrunde gegangen ... So hatte Poincaré freies Feld ...".

Klein und Poincaré korrespondierten 1881/82 miteinander nach dem „do ut des"-Prinzip [K III, 587-621]. Bald begehrte Klein gegen die Taufnamen ,groupe fuchsien, fonction fuchsienne" auf. Für ihn waren Riemann und Galois die Paten der Poincaréschen Theorie. „Fuchs hat hier keine Verdienste" [K1926, S. 374]. Schließlich war Poincaré Kleins Polemik leid. Am 4. April 1882 erinnert er Klein an das FaustWort „Name ist Schall und Rauch" und schreibt schlußendlich [K III, S. 611]: „Après tout, ça m'est égal, faites comme vous voudrez, je ferai comme je voudrai de mon côté." Kleins Schüler Adolf Hurwitz fühlte sich an ein Kinderlied erinnert; am 6. April 1882 schreibt er an Klein [H1882]: „Es macht [Fuchs] empfindlich, daß Sie singen: ,Fuchs, Du hast die Function gestohlen, gieb sie wieder her! “ Später setzte sich die Bezeichnung ,automorphe Funktion“ durch [K III, S. 549], hingegen sind Fuchssche und Kleinsche Gruppen Standardbegriffe geworden.

\section{Ikosaeder-Buch}

In seinem Rückblick auf das Schicksalsjahr 1882 scheut Klein sich nicht zu bekennen: „Das Zentrum meines produktiven Denkens war zerstört" [K III, S. 585]. In dieser Zeit schwerster Depressionen beginnt er, „um eine leichte Arbeit zu haben“ mit der Niederschrift seines berühmten Buches über das Ikosaeder. „Ich ... sah mich veranlaßst, meine Tätigkeit fortan auf ein anderes Niveau einzustellen ... Solcherweise sind zunächst die, Vorlesungen über das Ikosaeder und die Auflösung der Gleichungen fünften Grades' zustande gekommen, die Ostern 1884 bei B. G. Teubner als selbständiges Werk erschienen sind" [K II, S. 258]. Die Leidenschaft zum Mischen, zum Zusammenbiegen, verschiedene Disziplinen einander durchdringen zu lassen, ist ein hervorstechender Zug dieses Buches. Weyl nannte es „eine wunderbare Symphonie, in welcher Geometrie, Algebra, Funktionentheorie und Gruppentheorie zu einer vieltönenden, aber von tiefsten Zusammenhängen durchwalteten Melodie zusammenklingen" [W1930, S. 294]. Klein, von dem das Wort „Formeln sind blind“ stammt, scheut jetzt nicht einmal den „Schraubstock der Rechnung" und notiert sogar spröde numerische Gleichungen. Das Ziel ist die algebraischgeometrische Klärung der verschiedenen Verfahren zur Auflösung von Gleichungen fünften Grades, seine Vorbilder sind Brioschi, Hermite, Kronecker und natürlich Galois. Die ungebrochene Aktualität der Ikosaedermathematik dokumentiert die 1993 von P. Slodowy besorgte Neuauflage [K1884] des Ikosaederbuches mit vielen liebevollen Annotationen und Literaturhinweisen bis in die Gegenwart. 


\section{Klein und der Glaubenskrieg zwischen Berlin und Göttingen}

Schon in den sechziger Jahren gab es Spannungen zwischen den Weierstraß-Jüngern in Preußens Capitale und Riemanns Getreuen hier in der Provinz. In seiner Selbstbiographie [Koe1919], S. 54, schreibt Leo Koenigsberger (1837-1921) im Rückblick auf seine Berliner Jahre von 1857-1864: „Wir gaben [den Leistungen der Göttinger Schule] häufig nicht sogleich die Stelle, welche die Wissenschaft ihnen alsbald anwies." Bekanntlich hatte Weierstraß nach Erscheinen von Riemanns Abelschen Funktionen (1857) sein bei der Berliner Akademie schon angenommenes Manuskript über eben diese Funktionen zurückgezogen und nicht mehr veröffentlicht. Zum Schisma führten Berliner Dogmen wie: Es gibt in der Funktionentheorie nur Potenzreihen. Es gibt keine Riemannschen Flächen. Dagegen glaubten Häretiker wie Carl Gottfried Neumann (1832-1925), Friedrich Alfred Clebsch (1833-1872), Alexander Wilhelm von Brill (1842-1935), Max Noether (1844-1921), Gustav Roch (1839-1866), Friedrich Emil Prym (1841-1915), Paul Albert Gordan (1837-1912) und, last not least, Felix Klein fest an Riemanns Ideen.

Weierstraß mit seiner Integralphobie schätzte Riemanns Flächen gering, beteiligte sich aber nicht an der Polemik gegen Riemann selbst, den er laut Carl Runge (1856-1927), wie einen Bruder geliebt habe“ [Ru1926, S. 179]. Ganz anders stand er zu Klein. Dieser ging ,entgegen den Wünschen von Clebsch, der mir genau so wie früher Plücker von diesem Plan abgeraten hatte, im Herbst 1869 nach Berlin, wo eine ganz andere mathematische Richtung herrschte" [K1923, S. 15]. Nach eigenem Bekunden hörte er 1869/70,,aus Widerspruchsgeist kein Kolleg bei Weierstraf" [K1926, S. 284].

Klein war von seinen Lehrern Plücker (1801-1868) und Clebsch geprägt. Clebsch war in den sechziger Jahren der dynamische Mathematiker außerhalb Berlins (zunächst in Gießen, ab 1868 in Göttingen). Die 1868 gemeinsam mit Carl Neumann gegründeten $M a$ thematischen Annalen waren die Plattform, mit der Clebsch und seine Verbündeten das Berliner Journal für die reine und angewandte Mathematik herausforderten. Klein wurde 1873 Mitherausgeber und 1876 geschäftsführender Herausgeber der „Annalen“. Er kommentiert später [K1926, S. 297]: „Als Clebsch 1872 so plötzlich starb, waren wir . . . in starke persönliche Gegensätze mit einem großen Teil der übrigen Mathematiker ... verstrickt. .. . Das Mißtrauen gegen uns ging so weit, daß die Mathematischen Annalen, in denen wir ... unsere Arbeiten zu veröffentlichen pflegten, in Acht und Bann getan wurden."

In allerhöchste Ungnade fiel Klein 1885 beim Papst in
Berlin, als er nach seinem Wechsel von Leipzig nach Göttingen die Berufung von Sophus Lie nach Leipzig als seinen Nachfolger durchsetzte [K I, S. 412]. In einem Brief vom 20. Dezember 1885 an Hermann Amandus Schwarz (1843-1921) bricht es aus Weierstraß heraus: „Wäre Leipzig eine Preussische Universität, so würde ich mich verpflichtet halten, über ein so unerhörtes Vorgehen, wie es der Leipziger Facultät beliebt hat, das eine Beleidigung ist für alle jetzt im kräftigen Mannesalter stehenden deutschen Mathematiker, an der zuständigen Stelle ein offenes Wort zu reden... Ein schöner Anfang der neuen Aera, die unter Kleins Präsidentschaft beginnen soll! P. Dubois trifft doch zuweilen den Nagel auf den Kopf, er nannte vor Jahren schon das Trifolium Klein-Lie-Mayer „société thuriféraire“ [U1999, S. 41].

1892, als Klein Interesse an einem Lehrstuhl in Berlin zeigt, kommt es zum Eklat. Im Protokoll der Berufungskommission (Nachfolge Kronecker und Weierstraß) vom 22. Januar 1892 wird Klein als „Blender, Faiseur und Kompilator" beschimpft, weiter finden sich dort vernichtende Urteile wie „Klein nascht", „Das Ikosaeder-Buch ist eine Compilation von Schwarz und Fuchs im Feuilletonstil". Es wird Kleins „,verderbliches Vorgehen auf wissenschaftlichem Gebiet“ angeprangert. „Er arbeitet nicht um der Sache willen, sondern er schreibt Lehrbücher aus anderer Arbeit", vgl. [Bie1988, S. 151].

Kurz darauf wurden die Lehrstühle mit Frobenius und Schwarz besetzt. Klein blieb in Göttingen. Er holte 1895 Hilbert aus Königsberg. „Der beste, zukunftsreichste Kollege sollte neben ihm wirken, gleichviel ob der junge Baum den alten überschatten würde" [S. 1949, S. 290]. Hilbert holte 1902 Minkowski. Eine neue große Zeit Göttingens begann: Hilbert erlöste ab 1900 das Dirichletsche Prinzip aus dem Dornröschenschlaf, in den Weierstraß es versenkt hatte. Göttingen erlebte jetzt eine RiemannRenaissance; Weierstraß-Jünger priesen ohne große Resonanz bis weit in das 20. Jahrhundert hinein Potenzreihen als den allein selig machenden Weg.

Der Bauernjunge (country lad), der 1904 die Universität Göttingen bezog, erinnert sich 1944 an seine mathematische Jugend hier nach der Jahrhundertwende [W1944, S. 122]:

Hilbert and Minkowski were the real heroes of the great and brilliant period, ... Klein ruled over it like a distant god, 'divus Felix', from above the clouds.

Und in seiner Muttersprache sagt er in [W1930, S. 299]:

Die von Klein entfachte Flamme brauchte nicht in ängstlicher Tradition priesterlich gehütet zu werden; 
sie brennt ... auf der Esse ... der mathematischen Schmiede und tut die kleine, die große Arbeit des Tages. Sein Werk wirkt fort, sein Name wird nicht vergessen sein.

\section{Würdigungen}

Die Naturwissenschaften (Wochenzeitschrift für die Fortschritte der Naturwissenschaft, der Medizin und Technik, Verlag von Julius Springer, Berlin) gab am 25. April 1919 im siebenten Jahrgang ein Sonderheft „Felix Klein zur Feier seines siebzigsten Geburtstages" heraus mit folgenden acht Artikeln:

Fricke, R.: Felix Klein zum 25. April 1919, seinem siebzigsten Geburtstage, 275-280; Voß, A.: Felix Klein als junger Doktor, 280-287; Wirtinger, W.: Klein und die Mathematik der letzten fünfzig Jahre, 287-288; Schoenflies, A.: Klein und die nichteuklidische Geometrie, 288-297; Carathéodory, C.: Die Bedeutung des Erlanger Programms, 297-300; Sommerfeld, A.: Klein, Riemann und die mathematische Physik, 300-303; Timerding, H. E.: Felix Klein und die Reform des mathematischen Unterrichts, 303-307; Prandtl, L.: Felix Klein und die Förderung der angewandten Wissenschaften, 307-310.

In den Sitzungsberichten der Berliner Mathematischen Gesellschaft erschienen 1926 im Band 25 drei Nachrufe:

Lorey, W.: Felix Kleins Persönlichkeit und seine Bedeutung für den mathematischen Unterricht, 54-68; Hamel, G.: Felix Klein als Mathematiker, 69-80; Prandtl, L.: Klein und die angewandten Wissenschaften, 81-87.

Der Universitätsbund Göttingen veröffentlichte 1949 im Band 26 zur Feier des hundertsten Geburtstages von Klein drei Reden:

Kneser, H.: Felix Klein als Mathematiker, 1-6; Prandtl, L.: Felix Klein und die Anwendungen der Mathematik, 7-11; Lietzmann, W.: Felix Klein und der mathematische Unterricht, 1116.

Weitere Würdigungen bzw. Nachrufe:

[Be1961] Behnke, H:: Felix Klein und die heutige Mathematik, Math.-Phys. Sem. Ber. 7, 129-144 (1961)

[BuSch1973] Burau, W. und B. Schoeneberg: Klein, Christian Felix, Dict. Scient. Biography 7, 396-400 (1973)

[C1925] Courant, R.: Felix Klein, Die Naturwissenschaften 13, 765-772 (1925); auch in Jahr. Ber. DMV 34, 197-213 (1926) [C1926] Courant, R.: Felix Klein als wissenschaftlicher Führer, Nachr. Ges. Wiss. Göttingen, 1-8 (1925/26)

[Kn1949a] Kneser, H.: Felix Klein. Zu seinem 100. Geburtstage am 25. April 1949, Arch. Math. 1, 413-417 (1948/49)

[Kn1949b] Kneser, H.: Der Mathematiker Felix Klein, Naturwissenschaftliche Rundschau, Heft 5, 225-228 (1949)

[L1925] Lietzmann, W.: Erinnerungen an Felix Klein, Zeitschr. für math. u. naturwiss. Unterricht 56, 257-263 (1925)

[S. 1949] Sommerfeld, A.: Zum hundertsten Geburtstag von Felix Klein, Die Naturwissenschaften 36, 289-291 (1949)

[T1981] Tobies, R.: Felix Klein, Biographien hervorragender Naturwissenschaftler, Techniker und Mediziner 50, B. G. Teubner Verlagsgesellschaft, Leipzig 1981

[Wa1926] Walther, A.: Felix Klein, Zeitschr. technische Phys. 7, 2-7 (1926)

[W1930] Weyl, H.: Felix Kleins Stellung in der mathematischen Gegenwart, Die Naturwissenschaften 18, 4-11 (1930); Ges. Abh. III, 292-299

\section{Literatur}

[Bie1988] Biermann, K.-R.: Die Mathematik und ihre Dozenten an der Berliner Universität 1810-1933, Akad. Verlag Berlin 1988

[BN1894] Brill, A. und M. Noether: Die Entwicklung der Theorie der algebraischen Functionen in älterer und neuerer Zeit, Jahr. Ber. DMV 3, 107-566 (1894)

[D1975] Dieudonné, J.: Poincaré, Jules Henri, Dict. Scient. Biography 11, 51-61 (1975)

[H1882] Hurwitz, A.: Brief an Felix Klein vom 6. April 1882, Cod. Ms. F. Klein 9, Brief 913, Staats- und Universitätsbibliothek Göttingen

[K I-III] Klein, F.: Gesammelte Mathematische Abhandlungen, 3 Bände

[K1880/81] Klein, F.: Funktionentheorie in geometrischer Behandlungsweise, Vorlesung, gehalten in Leipzig 1880/81, ed. F. König, Teubner-Archiv zur Mathematik 7, Leipzig 1987 [K1884] Klein, F.: Vorlesungen über das Ikosaeder und die Auflösung der Gleichungen fünften Grades, Teubner Verlag 1884, neu herausgegeben und annotiert von P. Slodowy, Birkhäuser und Teubner Verlag 1993

[K1891/92] Klein, F.: Riemannsche Flächen, Vorlesungen, gehalten in Göttingen 1891/92, ed. G. Eisenreich und W. Purkert, Teubner-Archiv zur Mathematik 5, Leipzig 1986

[K1923] Klein, F.: Göttinger Professoren. Lebensbilder von eigener Hand, Mitteil. Universitätsbund Göttingen, 5. Jahrg., 11-36 (1923)

[K1926] Klein, F.: Vorlesungen über die Entwicklung der Mathematik im 19. Jahrhundert, Band 1, Springer Verlag 1926, Nachdruck 1979

[Koe1919] Koenigsberger, L.: Mein Leben, Carl-Winters Univ. Buchhandlung, Heidelberg 1919

[P I-XI] Poincaré, H.: CEuvres, 11 Bände

[Pa1997] Patterson, S.: Uniformisierung und diskontinuierliche Gruppen, in [W1913-97]

[R 1876-1990] Riemann, B.: Gesammelte Mathematische Werke und wissenschaftlicher Nachlass, herausgegeben unter Mitwirkung von R. Dedekind von H. Weber, Teubner Verlag Leipzig 1876; 2. Auflage 1892; Nachträge herausgegeben von M. Noether und W. Wirtinger 1902; Gesammelte Mathematische Werke, wissenschaftlicher Nachlass und Nachträge. Nach der Ausgabe von H. Weber und R. Dedekind neu herausgegeben von R. Narasimhan, Springer-Verlag/Teubner Verlag 1990 [Re1998] Remmert, R.: From Riemann surfaces to complex spaces, Matériaux pour l'Histoire des Mathématiques au XXe siècle, Actes du colloque à la mémoire de Jean Dieudonné, (Nice, 1996), Séminaire et Congrès 3, Soc. Math. de France, 203-241 (1998)

[Ro2000] Rowe, D. E.: Episodes in Berlin-Göttingen Rivalry, 1870-1930, Math. Intelligencer 22, Heft 1, 60-69 (2000)

[Ru1926] Runge, C.: Persönliche Erinnerungen an Karl Weierstraß, Jahr. Ber. DMV 35, 175-179 (1926)

[U1999] Ullrich, P.: Über die Wichtigkeit einer lesbaren Handschrift: Weierstraß's Poltern zur Berufung von Lie nach Leipzig, Mitt. DMV 4/1999, 39-42

[W1913-97] Weyl, H.: Die Idee der Riemannschen Fläche, Teubner Verlag Leipzig 1913; anastatischer Neudruck 1923, umgearbeitete 3. Auflage 1955. Nachdruck der 1. Auflage mit Annotationen, ed. R. Remmert, 1997

[W1944] Weyl, H.: Obituary: David Hilbert 1862-1943, Ges. Abh. IV, 121-129

[W1955] Weyl, H.: Rückblick auf Zürich aus dem Jahre 1930, Schweizerische Hochschulzeitung 28, 180-189 (1955); Ges. Abh. IV, 650-654

\section{Adresse des Autors}

Prof. Dr. Reinhold Remmert

Mathematisches Institut, Universität Münster

Einsteinstraße 62, 48149 Münster 